\title{
The Role of Clinical Prediction Scores in Thrombotic Thrombocytopenic Purpura
}

\author{
Sevoyan $A^{1}$, Chakhoyan $A^{1}$, Ghazaryan $P^{2 *}$, Ter Grigoryan $A^{1}$, Hakobyan $Y^{1}$, Grigoryan $H^{1}$, \\ Oseyan $H^{1}$ and Martirosyan $N^{1}$
}

${ }^{1}$ Department of Clinical Hematology, Hematology Center after R. H. Yeolyan, Republic of Armenia
${ }^{2}$ Scientific Director, Hematology Center after R. H. Yeolyan, Republic of Armenia

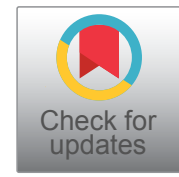

\section{Introduction}

Thrombotic thrombocytopenic purpura (TTP) is a fulminant and life-threatening blood disorder characterized by a pentad of microangiopathic hemolytic anemia, thrombocytopenia, acute renal failure, fever, neurological abnormalities, and multiple organ ischemias due to disseminated microvascular platelet-rich thrombi. This state is caused by a severe deficiency of ADAMTS13 protein (protease that cleaves von Willebrand factor (VWF) into smaller sizes and thereby eliminates unusually large VWF multimers that would otherwise accumulate on endothelial cells where they can cause platelet thrombi). TTP can be classified as congenital (extremely rare, genetic deficiency of the ADAMTS13 gene resulting in decreased or absent ADAMTS13 enzyme activity) and acquired (presence of an autoantibody against ADAMTS13 enzyme). Diagnosis of TTP is confirmed by the detection ADAMS13 activity, autoantibodies anti ADAMTS13 and screening for ADAMTS13 mutation.

Frequently the disease is misdiagnosed due to multivariate clinical symptoms. Sometimes there are difficulties in distinguishing TTP from hemolytic uremic syndrome. However during last years the progress was made in diagnostic process of TTP.

In Armenia the prevalence of TTP is 3 per 3.000 .000 population. Occurrence is 1 per million. The cases of TTP were underestimated in Armenia.

Patient, 44-year-old female presented with weakness, headache, vomiting, bruisees all over the body and uterine bleeding. The symptoms lasted for 10 days. At the time of presentation patients' consciousness was clear, slightly inhibited. Patient answered questions with delay and had no signs of focal neurological and meningeal impairment. No evidence of autoimmune diseases in the past. No history of cancer. Stool is normal. The CBC was the following HB-92 g/l, RBC-2.97 × 10 $12 / \mathrm{L}$, MCV-91.2 fl, MCH-31.0 pg, Ret.-130.9\%, PLT-9.0 $\times 10^{9} /$ I, WBC-10.01 $\times 10^{9} /$ l, Metam-1.0, banded neut6.0, Segment.-65.0, Lymph-19.0, Mon-7.0. Biochemistry test results revealed the high level of total bilirubin 65 $\mathrm{mmol} / \mathrm{l}$ mainly in a form of indirect bilirubin and lactate dehydrogenase (LDG). Creatinine was within normal ranges $0.8 \mathrm{mg} / \mathrm{dl}$. Tests for disseminated intravascular coagulation were negative. Fibrinogen was $3.58 \mathrm{~g} / \mathrm{I}$, INR-1.25. Test for HIV infection is negative. Direct Cooms test was negative. Ferritin level was $400 \mathrm{ng} / \mathrm{ml}$, Vitamin B12 was $263.9 \mathrm{pg} /$ $\mathrm{ml}$, Folic acid level-was $6.59 \mathrm{ng} / \mathrm{ml}$. The examinatiom of rheumatoid markers such as Lupus anticoagulant-1.02, Antithrombin-125.5\%, ANAs-negative, Anti-dsDNA IgG $<8.61$ $\mathrm{IU} / \mathrm{ml}$, Anti-beta 2 glycoprotein1-12.7 RU/ml, Anti-Cardiolipin antibodies $<1.2 \mathrm{U} / \mathrm{ml}$ did not reveal abnormalities. The morphological examination of the bone marrow describe the normal cellularity of bone marrow and increased number of megakaryocytes without platelet production. Examination for ADAMTS13 was not done due to absence of the mentioned test in Armenia. The function of thyroid gland was normal. USD examination shows the increase of liver sizes up to $17 \mathrm{~cm}$, no other abnormalities were detected. CT-scan of the brain did not reveal pathological changes. During investigation period neurological symptoms were worsening and the loss of consciousness was observed. There is classic pentad for TTP composing of clinical pathological signs: Thrombocytopenia, microangioopathic hemolytic anemia, neurological abnormalities, renal failure and fever. TTP does not often manifest with classic pentad. However the constant signs for TTP are thrombocytopenia $\left(<30 \times 10^{9} /\right.$ I) and microangiopathic hemolytic anemia. There are several clinical prediction scores like PLASMIC, FRENCH, BENTIEY scoring systems intended for use as clinical prediction scores aimed to enhance physician's clinical judgment and guide initial therapy management. PIASMIC score include low

* Corresponding author: Ghazaryan P, Scientific Director of Hematology center after R. H. Yeolyan. 0014, Yerevan, Republic of Armenia

Accepted: January 29, 2022

Published online: January 31, 2022

Citation: Sevoyan A, Chakhoyan A, Ghazaryan P, et al. (2022) The Role of Clinical Prediction Scores in Thrombotic Thrombocytopenic Purpura. Clin Hematol Res 5(1):62-63 
platelet count, indirect bilirubin level, reticulocytes count, INR, creatinine level and the absence of history of cancer and transplantation [1,2].

As the test for ADAMTS13 was not done the PLASMIC scoring system was used with the aim to evaluate the likelihood of ADAMTS13 deficiency. By calculating the criteria for this patient we registered 6 point that predicts the likelihood of ADAMTS13 deficiency is of $62 \%-82 \%$. Combination of clinical symptoms with the laboratory results: The high level of increased level of total bilirubin in a form of indirect bilirubin and LDG, low level of platelets and exclusion of other possible reasons of thrombocytopenia bring us to the establishment of diagnosis of acquired trombotic thrombocytopenic purpura.

Plasma infusion and plasma exchange therapy were done. 4 sessions of plasma exchange therapy were done. No significant improvement was observed. The treament with Rituximab was initiated. After 1st injection of Rituximab patient's consciousness became clear. Neurological symptoms and $\mathrm{CBC}$ parameters were slowly recovered. Treatment was continued with weekly rituximab injection (4 injections in total). The clinical response was achieved: sustained platelet count $210 \times 10^{9} /$, normal ranges of bilirubin and LDG and no clinical evidence of ischaemic episodes. Patient is in good clinical hematology condition under follow-up in Hematology Center.

\section{Discussion}

The examination of ADAMTS13 is mandatory criteria for the diagnosis of TTP, however in cases where test could not be done the combination of clinical and laboratory features and the use of clinical prediction scores could be crucial for the prompt establishment of diagnosis and immediate treatment for life threatening diseases like TTP.

\section{References}

1. Bendapudi PK, Hurwitz S, Fry A, et al. (2017) Derivation and external validation of the PLASMIC score for rapid assessment of adults with thrombotic microangiopathies: A cohort study. Lancet Haematol 4: e157-e164.

2. Tiscia GL, Ostuni A, Cascavilla N, et al. (2018) Validation of PLASMIC score and follow-up data in a cohort of patients with suspected microangiopathies from Southern Italy. J Thromb Thrombolysis 46: 174-179.

DOI: $10.36959 / 831 / 387$ 